\section{Not far out}

\section{Malcolm Longair}

The Farthest Things in the Universe. By Jay M. Pasachoff, Hyron Spinrad, Patrick S. Osmer and Edward Cheng. Cambridge University Press: 1994. Pp. 104. £19.95, $\$ 29.95$ (hbk); $£ 11.95$, \$19.95 (pbk).

THE dustjacket of this little book claims: "This survey offers an exceptional opportunity for the general audience to share the excitement of today's astronomical research of the early Universe in an accessible and stimulating way". These are encouraging words but one's enthusiasm is slightly dampened on reading the foreword. The book originates from a series of popular lectures delivered at a meeting of the American Association for the Advancement of Science in San Francisco in 1989. Two of the original lecturers, Patrick Osmer and Hyron Spinrad, have rewritten their contributions to bring them up to date on the topics of quasars and large-redshift radio galaxies respectively. Edward Cheng has written a completely new chapter on COBE observations of the cosmic microwave background radiation and Jay Pasachoff, the general editor, has provided an introductory chapter describing the large-scale structure of the Universe and how it is observed. Inevitably, there is a feeling of thinness about the material covered: one suspects that the original lectures provided a much more complete tour d'horizon of what is undoubtedly one of the most important areas of modern astrophysical cosmology.

The four chapters contain a lot of excellent material and the general reader can be reassured that, so far as they go, they are authoritative and the views expressed are sound. But there is a big difference between an exciting and stimulating lecture and the hard daylight of the printed word. There is no attempt to provide a systematic, coherent account of what is now known about the early Universe. The reader will look in vain for any discussion of primordial nucleosynthesis and the origin of the light elements, the problems of the origin of the formation of structure in the Universe, what the inflationary model of the early Universe is and why it is taken seriously, the physics of dark matter and why it is believed to be central to the cosmological problem, the contributions of radio, infrared, ultraviolet, X-ray and gamma-ray astronomy to studies of the Universe at large redshifts, and so on. Surely these topics are so relevant to current cosmological research that chapters on them should have been included.
On the positive side, the chapters make clear the central contribution of the new high technologies to recent astronomical achievements. The level of presentation of the material varies considerably throughout. Pasachoff and Cheng make every effort to communicate the essence of their topics in an accessible way but there remains the difficulty of describing physical processes in the very early Universe in a meaningful way to a lay audience. Osmer's article is written for the enthusiast who has already done some homework on quasars and Spinrad's article is more or less aimed at the professional. The general reader will find the going tougher and tougher as the story unfolds, and there is not the crutch of a glossary at the end to support the aspiring enthusiast.

It is crucial that experts in all areas of science reach out to the general public and describe in their own words the full significance of their research. Unfortunately, this book misses a real opportunity to do this. Those who want authoritative reviews of the specific areas discussed will not be disappointed. But those wanting a broader perspective of the key issues of modern astrophysical cosmology will have to look elsewhere.

Malcolm Longair is in the Cavendish Laboratory, University of Cambridge, Madingley Road, Cambridge CB3 OHE, UK.

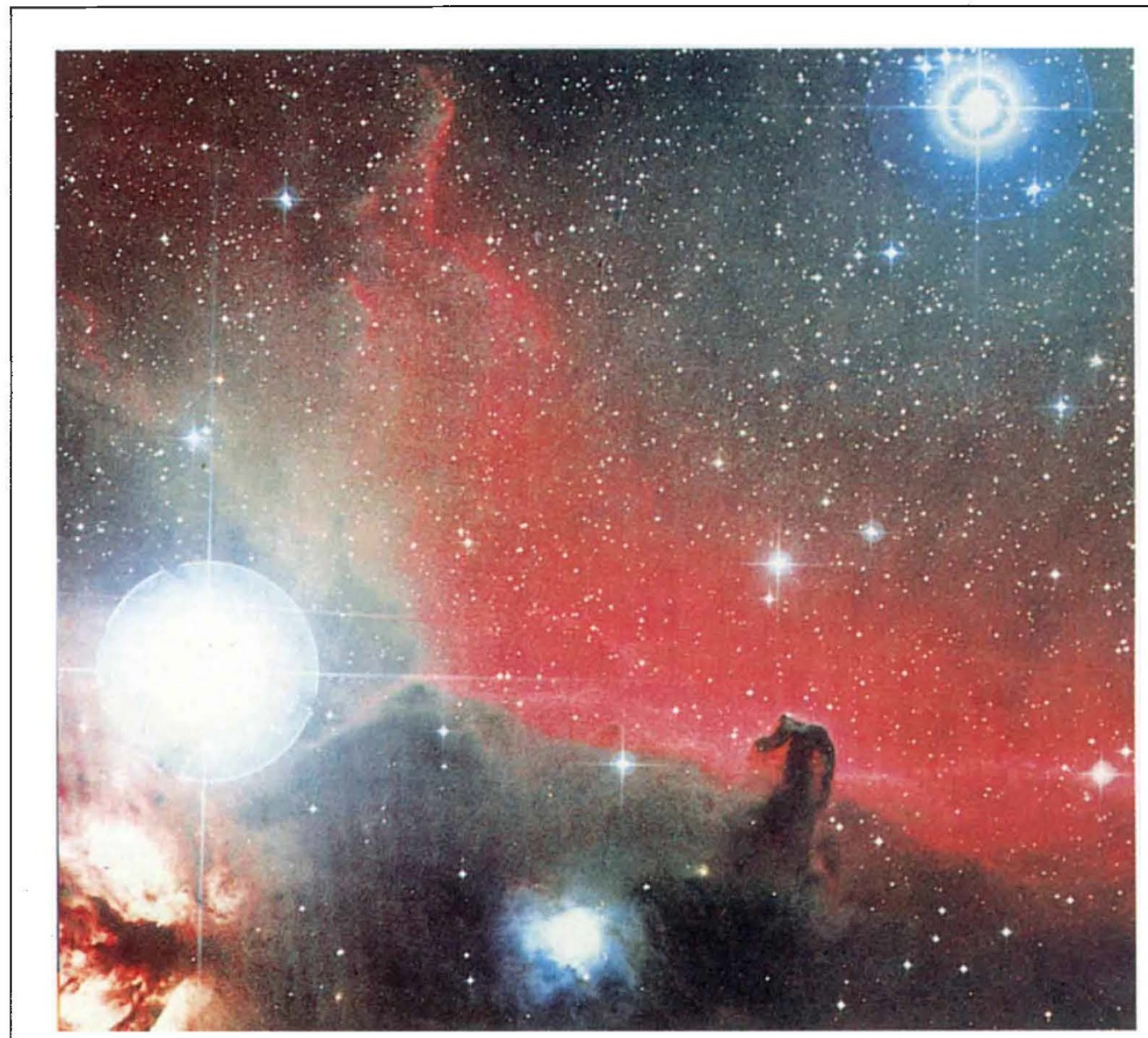

THIS famous photograph by David Malin of Orion's Horsehead Nebula, a dense dark cloud where dust is very abundant and which completely absorbs the light from regions behind it, adorns the cover of the third edition of The Cambridge Atlas of Astronomy edited by J. Audouze, G. Israel and J.-C. Falque (Cambridge University Press, pp. 471, $\$ 50, \$ 75)$. Well-organized, richly illustrated and superbly presented, this large-format guide to planetary science, astronomy and astrophysics has been extensively revised and updated. It now includes material on the Magellan mission, new sections on Neptune, Pluto and solarsystem debris, and a 24-page glossary and index. 